\title{
Percepción de parámetros estéticos de la sonrisa en Odontólogos, estudiantes de odontología y pacientes de la Universidad Mayor de Santiago, Chile
}

\section{Perception of aesthetic parameters of the smile in dentists, dental students and patients of the University Mayor of Santiago, Chile}

\author{
S. Bofill Fontboté* , F. Williamson Dargham**, K. Cornejo Urzúa***, G. Letelier Sepúlveda***
}

\section{RESUMEN}

Introducción: El análisis de la sonrisa en Odontología es fundamental al efectuar un tratamiento estético dentario. El propósito de este estudio es evaluar la percepción de distintos parámetros estéticos de la sonrisa entre Odontólogos, estudiantes de odontología y pacientes.

Materiales y métodos: Odontólogos, estudiantes de odontología y pacientes evaluaron 5 sets de fotografías de sonrisas a través de encuestas calificadas. Las fotografías fueron agrupadas de acuerdo a modificaciones efectuadas en 3 parámetros estéticos de la sonrisa: contorno gingival, línea labial (subdividida en A y B), y proporción dentaria (subdividida en ancho y largo). Los datos se analizaron mediante Chi-cuadrado. Resultados: Un $80 \%$ de los Odontólogos y estudiantes consideran más atractivo un contorno gingival simétrico, un 73\% de los pacientes consideran más atractivo hasta $1 \mathrm{~mm}$ de discrepancia del contorno gingival. Con respecto a la Línea labial A, tanto Odontólogos (67\%) como estudiantes (77\%) encontraron más atractiva una sonrisa con $2 \mathrm{~mm}$ de exposición gingival, $67 \%$ de los pacientes optaron $1 \mathrm{~mm}$ de exposición gingival. Para Línea labial B, $1 \mathrm{~mm}$ de exposición gingival fue considerado más atractivo por todos los encuestados. La proporción dentaria considerada mas atractiva entre el ancho del incisivo central y lateral fue $71 \%$, y para ancho/largo del incisivo central fue más atractiva una proporción del $75 \%$ esto para los tres grupos encuestados.

Discusión: El contorno gingival fue el parámetro más sensible al momento de evaluar la estética de la sonrisa, para los otros dos parámetros no se encontraron diferencias significativas en las percepciones.

PALABRAS CLAVE: Estética, percepción, contorno gingival, línea labial, proporción dentaria, sonrisa, atractivo, parámetro.
ABSTRACT
Introduction: Smile analisis in dentistry is essential when performin dental aesthetic treatments. The pur- pose of this study is to evaluate the perception of different aesthetic parameters of the smile among den- tists, dental students and patients.
Materials and methods: Dentists, dental students and patients evaluated 5 sets of smile pictures through
* $\quad$ Cirujano-Dentista. Profesora Asociada. Asignatura Odontología integral del adulto. Facultad de Ciencias. Escuela de Odontología. Universidad Mayor. Santiago, Chile.
** Cirujano-Dentista. Profesor Asociado. Asignatura Histología. Facultad de Ciencias. Escuela de Odontología. Universidad Mayor. Santiago, Chile.
*** Cirujano-Dentista. Facultad de Ciencias. Escuela de Odontología. Universidad Mayor. Santiago, Chile. 
qualified surveys. The photographs were grouped according to modifications made in 3 smile aesthetic parameters: gingival contour, lip line (subdivided into A and B), and dental proportion (further subdivided in width and length). Data was statistically analyzed using Chi-square test.

Results: $80 \%$ of dentists and students considered more attractive a symmetrical gingival contour, $73 \%$ of patients consider more attractive a gingival contour discrepancy up to $1 \mathrm{~mm}$. Regarding Lip Line A, both Dentists (67\%) and students (77\%) found more attractive a smile with $2 \mathrm{~mm}$ of gingival exposure, $67 \%$ of patients prefered $1 \mathrm{~mm}$ of gingival exposure. Lip Line B, with $1 \mathrm{~mm}$ of gingival exposure, was considered more attractive by all respondents. The dental proportion between the width of the central and lateral incisors considered more attractive was $71 \%$; for the width / length of the central incisors, a proportion of $75 \%$ was more attractive for the all the groups surveyed.

Discussion: Gingival contour was the most sensitive parameter when evaluating the aesthetics of the smile. No significant diffrences in perception were found for the other two parameters included in this study.

KEY WORDS: Aesthetics, perception, gingival contour, labial line, dental proportion, smile, attractiveness, parameter.

Fecha de recepción: 13 de diciembre de 2019

Fecha de aceptación: 15 de enero de 2020

S. Bofill Fontboté, F. Williamson Dargham, K. Cornejo Urzúa, G. Letelier Sepúlveda. Percepción de parámetros estéticos de la sonrisa en Odontólogos, estudiantes de odontología y pacientes de la Universidad Mayor de Santiago, Chile. 2020; 36, (4): 191-199.

\section{INTRODUCCIÓN}

La estética de la sonrisa no solo es indispensable para el atractivo facial, sino que es un importante factor que contribuye al bienestar biopsicosocial del individuo ${ }^{[1,2,3]}$.El análisis de la sonrisa es parte importante de la evaluación estética facial general que diariamente realizan los Odontólogos ${ }^{[4,5]}$.

Una sonrisa agradable dependerá de la armonía entre los diferentes parámetros que definen la estética de ésta, los cuales deben ser considerados al momento de realizar una rehabilitación oral, de manera de dar respuesta a la necesidad cada vez mayor de los pacientes de obtener una sonrisa atractiva ${ }^{[5,6]}$. En este estudio se analizaron los siguientes parámetros estéticos: 1) Armonía del contorno gingival, 2) Línea Labialy 3) Proporción Dentaria.

La armonía en el contorno gingival es fundamental al momento de rehabilitar, la altura gingival y la posición del zenit deben ser simétricos entre ambos centrales, laterales y $\operatorname{caninos}^{[7,8]}$. La modificación o variación de la posición del contorno gingival afectará el resultado estético de la sonrisa $^{[5,9,10,11]}$.

La línea labial relaciona el borde inferior del la- bio superior con las piezas dentarias anteriores y tejido gingival, se visualiza como la cantidad de tejido gingival que se despliega al sonreír ${ }^{[9,12]}$. Esta líneapermite clasificar en 3 tipos la sonrisa: alta, media o baja ${ }^{[13]}$.

La proporción entre el ancho y el largo del incisivo central se define como la relación porcentual entre la distancia mesio-distal de la corona en su superficie vestibular más ancha y su longitud medida desde el borde incisal al margen gingival ${ }^{[9][14][15]}$.

Determinar la preferencia en los estándares estéticos de tales factores proporciona una guía en la planificación y tratamiento .La percepción de los parámetros estéticos juega un rol fundamental en la obtención de una sonrisa armónica, este estudio tiene como objetivo evaluar la percepción de los tres parámetros estéticos de la sonrisa antes mencionados entre odontólogos, estudiantes de odontología y pacientes, y de esta forma contribuir a una mayor satisfacción en el tratamiento estético de los pacientes.

\section{MATERIALES Y MÉTODOS}

En este estudio se utilizó una muestra total de 90 sujetos, 30 odontólogos, 30 estudiantes de odon- 
tología y 30 pacientes, elegidos por conveniencia. Dentro de los parámetros estéticos, las variables analizadas fueron: 1) Armonía del contorno gingival, 2) Línea labial, 3) Proporción dentaria del incisivo central e incisivo lateral superior.

Los criterios de inclusión fueron; odontólogos y estudiantes de la Escuela de Odontología de la Universidad Mayor y pacientes atendidos en la Clínica de integral del adulto durante el año 2018, que estuvieron dispuestos a participar en el estudio. Los criterios de exclusión fueron, pacientes con alguna alteración visual severa que impidiera una correcta evaluación de las fotografías (disminución del campo visual, cirugía ocular reciente), Odontólogos con menos de 3 años de experiencia laboral, y personas que no quisieron participar en el estudio.

Se tomaron fotografías estandarizadas del tercio inferior del rostro de una persona de género femenino, entre 20 y 30 años, mostrando una sonrisa sin restauraciones o tratamientos estéticos previos en el sector dentario anterior y con una proporción dentaria de acuerdo con el promedio. Se le realizó un formulario de consentimiento informado a la candidata para manipular digitalmente su sonrisa y utilizarla en este estudio. Se utilizó una cámara réflex Nikon D5600 de 24 megapíxeles con Lente $18-55 \mathrm{~mm}$, diafragma 3,5-5,6.

Las fotografías fueron capturadas sólo por un operador calibrado para este efecto, y se estudiaron digitalmente con el software de edición de fotografías Adobe ${ }^{\circledR}$ Photoshop ${ }^{\circledR}$ CC para realizar las modificaciones de los parámetros estéticos establecidos en el estudio; cabe resaltar que los parámetros se modificaron de uno a la vez, realizando sets de fotografías de cada parámetro estético para analizar
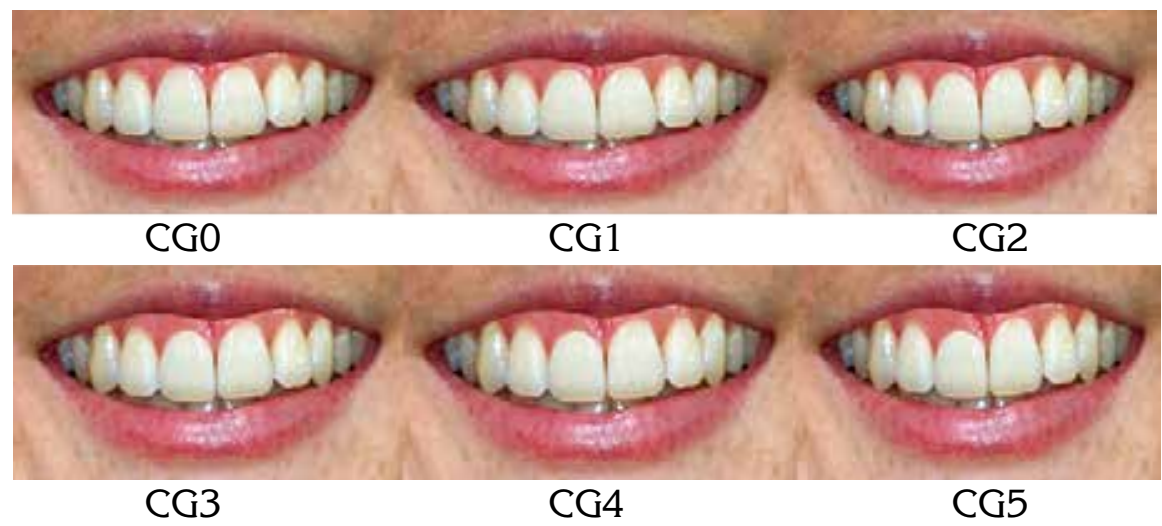

Figura 1. Set de imágenes de contorno gingival, distintas discrepancias en los márgenes de los incisivos centrales. CG0 $=0 \mathrm{~mm}, \mathrm{CG} 1=0,5 \mathrm{~mm}, \mathrm{CG} 2=1 \mathrm{~mm}, \mathrm{CG} 3$ $=1,5 \mathrm{~mm}, \mathrm{CG} 4=2 \mathrm{~mm}, \mathrm{CG} 5=2,5 \mathrm{~mm}$.

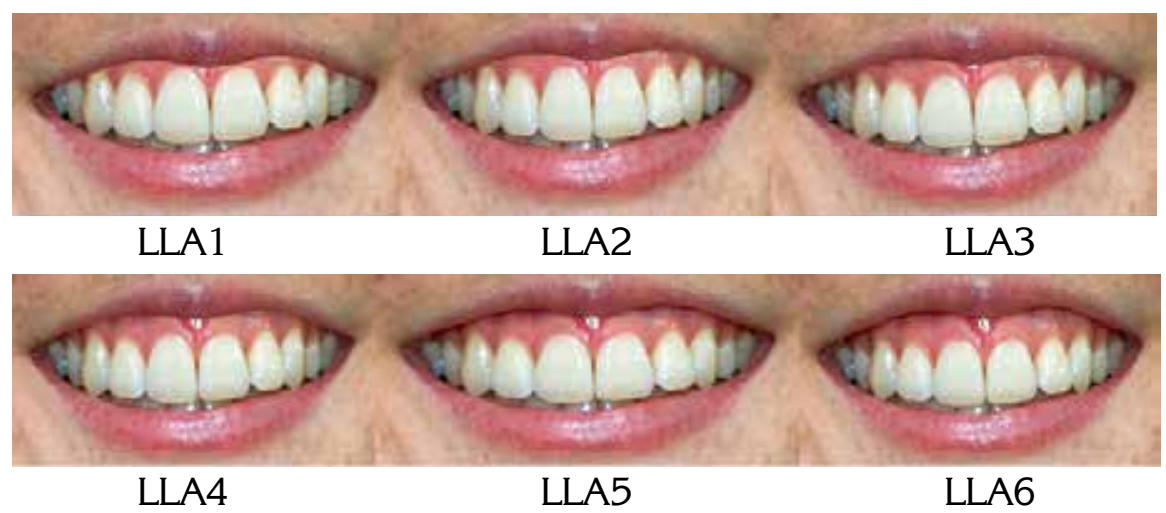

Figura 2. Set de imágenes para línea labial A, evaluando mm de exposición gingival. LLA1 $=1 \mathrm{~mm}, \mathrm{LLA} 2=2 \mathrm{~mm}, \mathrm{LLA} 3=3 \mathrm{~mm}, \mathrm{LLA} 4=4 \mathrm{~mm}, \mathrm{LLA} 5=5 \mathrm{~mm}, \mathrm{LLA} 6=6 \mathrm{~mm}$.

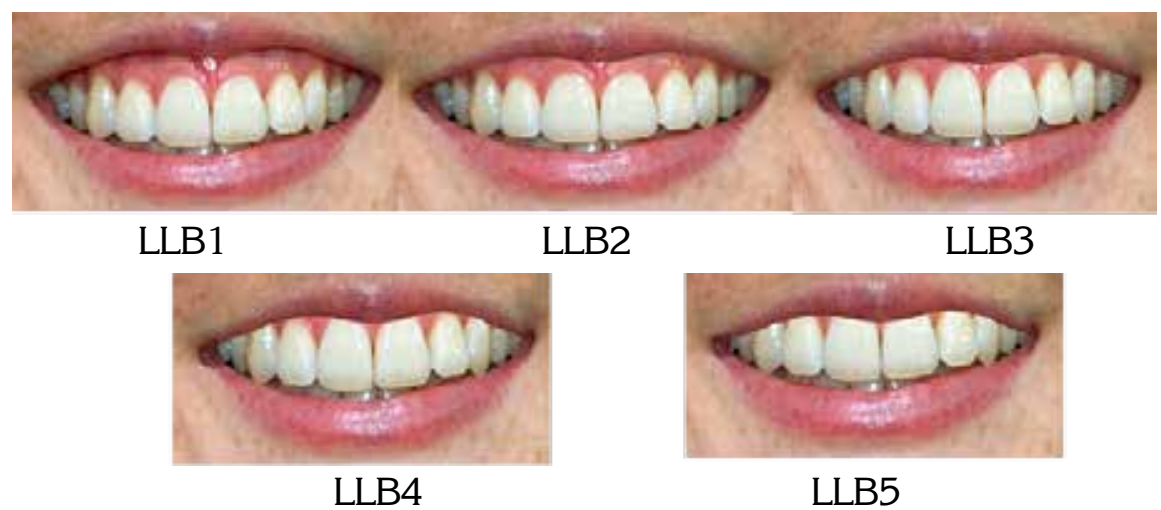

Figura 3. Set de imágenes para línea labial $\mathrm{B}$, evaluando distintas posiciones del labio superior al sonreír. $L L B 1=5 \mathrm{~mm}$ apical a incisivos superiores, $L L B 2=3 \mathrm{~mm}$ apical a incisivos superiores, $L L B 3=1 \mathrm{~mm}$ apical a incisivos superiores, $L L B 4=1 \mathrm{~mm}$ cubriendo la corona de incisivos superiores y LLB5 $=3 \mathrm{~mm}$ cubriendo la corona de los incisivos superiores.

la percepción a la modificación de éstos.

Para el primer parámetro correspondiente a la armonía del contorno gingival (CG), se modificó la altura del margen gingival en una escala 
de $0,5 \mathrm{~mm}$ en el incisivo central superior derecho, desde $0 \mathrm{~mm}$ hasta $2,5 \mathrm{~mm}$ de discrepancia entre los incisivos centrales superiores, resultando 6 fotografías como se observa en la figura 1.

El segundo parámetro corresponde a la Linea Labial la cual se subdividió en dos grupos, 1) Linea Labial A (LLA) donde se evaluó sólo la exposición gingival, utilizando una escala de $1 \mathrm{~mm}$, desde $1 \mathrm{~mm}$ sobre los incisivos centralessuperiores hasta $6 \mathrm{~mm}$, resultando 6 fotografías.(figura 2) y en el grupo 2) Línea Labial $B(L L B)$ se evaluó la línea labial, pasando de alta a baja, en una escala de $2 \mathrm{~mm}$, desde 5 $\mathrm{mm}$ de exposición gingival hasta 3 $\mathrm{mm}$ cubriendo las coronas de los incisivos superiores, resultando 5 fotografías (figura 3).

El tercer parámetro modificado fue la proporción dentaria, del cual se analizaron dos grupos: Grupo $\mathrm{N}^{\circ} 1$ : La proporción del ancho entre incisivo central y lateral superior derecho (PA), resultando 6 fotografías, en las que se modificó el

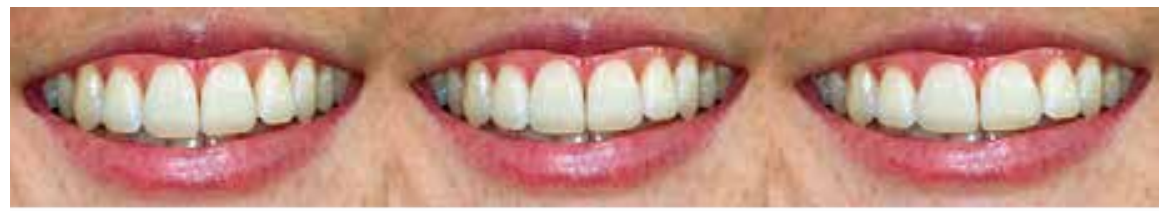

PA1

PA2

PA

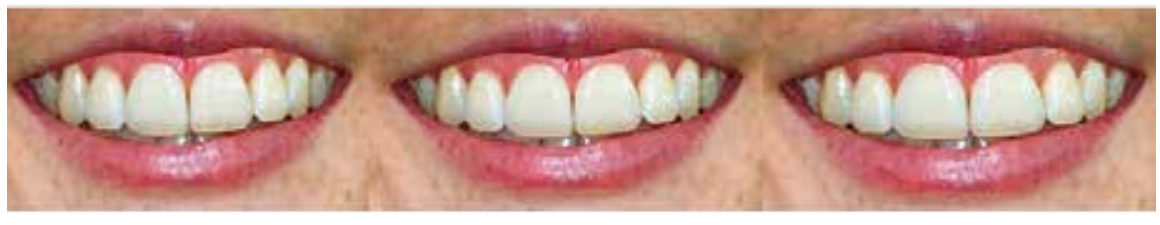

PA4

PA5

PA6

Figura 4. Set de imagen para la proporción del ancho entre incisivo central y lateral superior derecho. Evaluando diferentes proporciones. PA $1=71 \%$, PA $2=67 \%$, PA3 $=65 \%$, PA4 $=62 \%$, PA5 $=59 \%$, PA6 $=57 \%$.

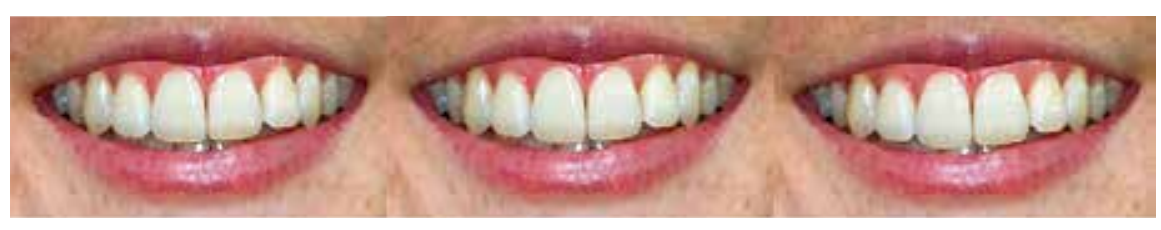

PL1

PL2

PL3

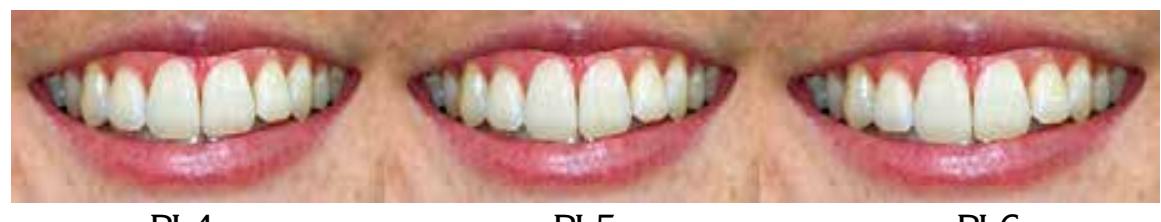

PL4

PL5

PL6

Figura 5. Set de imagen para la proporción del ancho-largo del incisivo central. Cada imagen representa una proporción diferente entre el ancho-largo del incisivo central. PL1 $=75 \%$, PL2 $=72 \%$, PL3 $=70 \%$, PL4 $=68 \%$, PL $5=66 \%$, PL6 $=64$ ancho del incisivo central superior derecho en los puntos de contacto mesial y distal más alejados de la corona en su superficie vestibular. Los incrementos fueron en rangos de $5 \%$ para producir cinco variaciones adicionales a la fotografía control alcanzando el 25\%,(figura 4). En este set de fotografías no existió variación en la posición del borde incisal, el plano incisal, el plano gingival y la longitud del incisivo central para poder calcular la proporción áurea definida por Lombardi ${ }^{[7,16,17]}$. En el Grupo $\mathrm{N}^{\circ} 2$ : La proporción ancho-largo del incisivo central superior derecho $(P L)$, con un set de 6 fotografías, se modificó el largo del incisivo central superior derecho, realizando incrementos de $0,5 \mathrm{~mm}$, esto se modificó cinco veces alcanzando un aumento de $2,5 \mathrm{~mm}$, ver figura 5 .

A cada fotografía se le agregó un código que fue utilizado para distinguir cada variación de un parámetro estético, y que posteriormente se utilizó para el análisis de resultados.
A la muestra se le expuso de forma digital las fotografías, a la altura de su vista, con una cantidad de brillo y un fondo estandarizado. Las fotografías se mostraron sin orden secuencial de los parámetros, para disminuir sesgos y no inducir visualmente la respuesta. Se realizó una encuesta en la cual las personas calificaron las imágenes como "atractiva" o "no atractiva", cabe señalar que la participación de las personas encuestadas en el estudio fue de manera voluntaria.

El test estadístico utilizado fue Chi cuadrado y se consideró una diferencia estadísticamente significativa de $\mathrm{p}<0,05$ utilizando el Software estadístico Stata v15.

\section{RESULTADOS}

Se realizó el análisis estadístico con las fotografías agrupadas con sus respectivos códigos en 5 
grupos ya definidos para analizar los parámetros estéticos de la investigación.

Se compararon las respuestas "atractivo" y "no atractivo" entre odontólogos, estudiantes de odontología y pacientes en cada uno de los grupos de parámetros estéticos. Las respuestas fueron separadas además por género, sin embargo, al comparar entre hombres y mujeres no se encontraron diferencias estadísticamente significativas, excepto en hombres del grupo contorno gingival, por lo que no se analizará en los resultados.

Se debe entender que sobre un 50\% de aceptabilidad en los gráficos, la variación se considera atractiva.

\section{Contorno Gingival}

En el grupo de Contorno Gingival, los odontólogos consideraron sólo atractivo y aceptable CG0, el cual fue nuestro control del parámetro contorno gingival, donde los márgenes de los incisivos centrales superiores están a la misma altura, considerándolo atractivo el $80 \%$ de los casos.

Los estudiantes también encontraron más atractivo CG0 en un $80 \%$, pero además consideraron estética hasta una diferencia de $0,5 \mathrm{~mm}$ (CG1) en los contornos gingivales. Los pacientes encontraron aceptables las variaciones de hasta $1 \mathrm{~mm}$ de discrepancia entre los márgenes de los incisivos centrales superiores (CG0, CG1, CG2), siendo la más atractiva para ellos $1 \mathrm{~mm}$ (CG2) en un $73 \%$ (Ver figura 6).

Fueron analizadas además, todas las respuestas para "atractiva" y "no atractiva" por separado. Para la respuesta "atractiva" existieron diferencias estadísticamente significativas $(\mathrm{p}=0)$, no así para la respuesta "no atractiva" $(\mathrm{p}=0,32)$.

\section{Línea LaBial A}

En el grupo de Línea Labial A, el 67\% de los odontólogos consideraron más atractiva una sonrisa cuando hay $2 \mathrm{~mm}$ de exposición gingival (LLA2) siendo la única aceptada por ellos. Los estudiantes consideraron aceptable cuando hubo hasta 3 $\mathrm{mm}$ de exposición gingival al sonreír, siendo la más atractiva en un $77 \%$ de los encuestados la exposición de $2 \mathrm{~mm}$ de encía (LLA2).Los pacientes encontraron más atractiva la sonrisa cuando se expuso $1 \mathrm{~mm}$ de encía (LLA 1 ) en un $67 \%$, siendo aceptable hasta $2 \mathrm{~mm}$ (LLA2). Ver figura 6.

Fueron analizadas las respuestas "atractiva" y "no atractiva" por separado, de todas las personas encuestadas. No se encontraron diferencias estadísticamente significativas para la respuesta "atractiva" $(p=0,86)$, y "no atractiva" $(p=0,97)$.

\section{LÍNEA LABIAL B}

En el grupo de Linea Labial B, el 70\% de los odontólogos y el $87 \%$ de los estudiantes consideraron más atractivo $1 \mathrm{~mm}$ de exposición gingival (LLB3), siendo aceptado hasta $3 \mathrm{~mm}$ (LLB2) con un $53 \%$ y $77 \%$ respectivamente.

Los pacientes consideraron un rango aceptable desde $3 \mathrm{~mm}$ de exposición gingival hasta $1 \mathrm{~mm}$ de cobertura de los incisivos centrales (LLB2, LLB3, LLB4), siendo más atractiva la sonrisa con 
$1 \mathrm{~mm}$ de exposición gingival (LLB3) con un $77 \%$. Ver figura 6.

En el análisis de todas respuestas para "atractiva" $(p=0,51)$ y "no atractiva" $(p=0,48)$, por separado, no se encontraron diferencias estadísticamente significativas.

PROPORCIÓN ENTRE EL ANCHO DEL INCISIVO CENTRAL Y LATERAL

En los tres grupos encuestados, fue considerada más atractivay aceptada una proporción dentaria de $71 \%$ (PA1), con un $83,3 \%$ los odontólogos, un93,3\% los estudiantes y un $80 \%$ los pacientes. La segunda proporción más aceptada por todos fue $67 \%$.

La proporción de $65 \%$, fue la última percibida de forma atractiva por odontólogos con un $56,7 \%$, estudiantes un $76,7 \%$ y pacientes un $63,3 \%$.

La proporción que encontraron menos atractiva fue $57 \%$, la cual fue seleccionada como atractiva solo por el 3,3\% de los odontólogos, $16,7 \%$ de los estudiantes y $26,7 \%$ de los pacientes. (Ver figura 6 )

En este parámetro no existieron diferencias estadísticamente significativas para las respuestas "atractiva" ( $p=0,127)$ y "no atractiva" $(p=0,127)$ entre odontólogos, estudiantes y pacientes.

PROPORCIÓN ENTRE EL ANCHO Y LARGO DEL INCISIVO CENTRAL

Fue considerada más atractiva la proporción $75 \%$ (PL1), de igual forma para odontólogos, estudiantes y pacientes, con $83,3 \%$ en los tres grupos.

Los odontólogos encontraron aceptablesólo hasta la proporción $72 \%$ (PL2). Los estudiantes aceptaron hasta la proporción 70\% (PL3) al igual que los pacientes.

El 96,7\% de odontólogos y estudiantes consideraron la proporción $66 \%$ (PL5) como poco atractiva,resultando la proporción menos atractiva en estos dos grupos, al igual que para los pacientes con un $76,7 \%$. (Ver figura 6)

No existieron diferencias estadísticamente significativas en el análisis de todas las respuestas para "atractiva" $(p=0,064)$ y "no atractiva" $(p=0,990)$.

\section{DISCUSIÓN}

En el parámetro de contorno gingival, los odontólogos percibieron de inmediato los cambios no aceptando discrepancias en este $(0 \mathrm{~mm})$, a diferencia de los estudiantes $(0.5 \mathrm{~mm})$ y pacientes $(1$ $\mathrm{mm}$ ) quienes sí aceptaron mayores discrepancias entre los márgenes. Existieron diferencias estadísticamente significativas en las respuestas "atractivo" para este parámetro.

En este estudio, al igual que en el de Al Taki A, et al., y Seoun $\mathrm{Mu}$ An, et al., se determinó que los odontólogos son los primeros en percibir mínimas variaciones en el contorno gingival ${ }^{[5,10]}$, por lo tanto, éstos tienen mayor agudeza visual percibiendo pequeños cambios del contorno gingival, pero cabe señalar que los estudiantes, al estar en un proceso de aprendizaje, poseen mayor discriminación que los pacientes, quienes perciben discrepancias más evidentes, siendo menos exigentes al momento de evaluar la estética de la sonrisa.

Este punto se debe tener en consideración al momento de rehabilitar a un paciente en el sector anterosuperior. Los odontólogos en ocasiones al percibir mínimas discrepancias en los márgenes de los incisivos centrales superiores buscan tratamientos, como cirugías de armonización del contorno gingival, creando necesidades en los pacientes que no son consideradas como tales por ellos. Este estudio demuestra la importancia de valorar la opinión del paciente y sus expectativas con respecto a los resultados estéticos del tratamiento.

En el parámetro Línea Labial A, los odontólogos coincidieron que $2 \mathrm{~mm}$ de exposición gingival era lo más atractivo, los pacientes por su lado consideraron más atractiva una exposición gingival de $1 \mathrm{~mm}$, sin embargo, ambos consideraron aceptable hasta $2 \mathrm{~mm}$, concordando con los resultados de Davis NC et al., y Kokich VO, et al., ${ }^{[12,}{ }^{18]}$ pero discrepando de los datos de Abu Alhaija ES, et al., que consideraron poco atractivo a partir de los 2 $\mathrm{mm}^{[4]}$. Los estudiantes difirieron encontrando que $2 \mathrm{~mm}$ de exposición gingival era lo más atractivo, y su umbral de aceptación fue mayor, tolerando hasta $3 \mathrm{~mm}$ de exposición.

Un rango de $1 \mathrm{~mm}$ hasta $3 \mathrm{~mm}$ de exposición gingival fue el aceptado para este estudio, siendo menor al rango mencionado en la revisión siste- 
mática realizada por Del Monte $\mathrm{S}$, et al., el cual se extiende desde 4,5 mm hasta 0,4 $\mathrm{mm}$ de exposición gingival ${ }^{[9]}$. Sin embargo, los datos obtenidos en este estudio coincidieron con los resultados descritos por la mayoría de los autoresy se puede considerar un rango control en las rehabilitaciones.Para futuras investigaciones, se podrían incluir mayores variaciones de exposición gingival, con valores intermedios de los datos utilizados en este estudio para analizar si existe discriminación para variaciones más pequeñas en la línea labial.

En el parámetro Línea Labial B, para todos los individuos encuestados fue más atractiva una línea labial con $1 \mathrm{~mm}$ de exposición gingival y menos atractiva cuando la exposición gingival fue mayor a $3 \mathrm{~mm}$. Tanto los odontólogos, estudiantes y los pacientes aceptaron una sonrisa hasta con $3 \mathrm{~mm}$ de exposición de encía, pero estos últimos también aceptaron cuando el labio cubría $1 \mathrm{~mm}$ la corona de los incisivos centrales, acercándose a los resultados obtenidos por Del Monte $\mathrm{S}$, et al., y Geron $\mathrm{S}$, et al., quienes encontraron aceptación de una línea labial $0,8 \mathrm{~mm}$ y $0,5 \mathrm{~mm}$ incisal al margen de los incisivos centrales respectivamente ${ }^{[9,19]}$. Sin embargo, todos los individuos de este estudio consideraron más atractivo cuando existía exposición gingival en la sonrisa, por lo que se consideró un rango aceptable desde $3 \mathrm{~mm}$ de exposición gingival hasta una línea labial de $1 \mathrm{~mm}$ incisal a los márgenes de los incisivos centrales superiores.

Si comparamos entre los parámetros Línea Labial $A$ y Línea Labial $B$, en ambos se expusieron imágenes de sonrisa con exposición gingival. Al comparar las respuestas de los estudiantes, coincidió el nivel de aceptación hasta $3 \mathrm{~mm}$ de visualización gingival, pero en cuanto a los odontólogos y los pacientes no existió coincidencia en las respuestas con respecto al nivel de aceptación, ya que para Línea Labial $A$ aceptaron hasta $2 \mathrm{~mm}$ y para Línea Labial $B$ hasta $3 \mathrm{~mm}$ de exposición gingival, esto probablemente se produjo porque en el grupo Línea Labial $B$ no existió la opción de $2 \mathrm{~mm}$ de exposición gingival, por ende la sonrisa más parecida fue aquella con $3 \mathrm{~mm}$ de visualización gingival, que es hasta donde consideraron aceptable en el grupo Línea Labial B.Los resultados obtenidos para Línea Labial en esta investigación no presentaron diferencias estadísticamente significativas entre Odontólogos, estudiantes y pacientes, por lo tanto, no es un parámetro tan sensible al momento rehabilitar la zona dentaria estética como lo es el contorno gingival.

En el parámetro de proporción dentaria entre el ancho del incisivo central y lateral superior, la proporción considerada más atractiva tanto para odontólogos, estudiantes y pacientes fue de $71 \%$, resultados similares fueron encontrados en el estudio de Al TakiAmjad, et al., quienes mostraron que tanto los odontólogos como los pacientes consideraron más atractiva aquella sonrisa en que la proporción entre el ancho del incisivo central y lateral fue de $67 \%^{[20]}$.

Además, en esta investigación, fueron consideradas aceptables las proporciones $71 \%$, 67\% y $65 \%$ tanto por odontólogos, estudiantes como pacientes, estos resultados están dentro del rango de las proporciones consideradas como atractivas en la revisión bibliográfica de Stefano Del Monte, et al., $y$ en el estudio similar al nuestro de Wolfart $S$, et al., los cuales consideran aceptable las proporciones entre $50 \%$ a $74 \% .^{[9,21]}$ En este estudio los tres grupos encuestados determinaron las mismas proporciones como aceptables, en contraste con los estudios de Wolfart S, et al., y Alsulaimani $\mathrm{FF}$, et al., en los cuales los pacientes presentaban aceptación de una mayor cantidad de proporciones a diferencia de los odontólogos ${ }^{[21,22]}$.

No se encontró diferencia estadísticamente significativa en las proporciones consideradas atractivas $(p=0,127)$ entre odontólogos, estudiantes y pacientes, concordando con los resultados propuestos por Al TakiAmjad, et al., el que indica que no presenta diferencia estadísticamente significativa para la sonrisa atractiva en este parámetro entre odontólogos y pacientes ${ }^{[20]}$.

Otro punto a discutir es la proporción áurea propuesta por Lombardi y Levin, la proporción entre el ancho del incisivo central e incisivo lateral superior deben ser del $62 \%{ }^{[12,20,22]}$. En el presente estudio esta proporción áurea propuesta fue aceptada por un bajo porcentaje de odontólogos, estudiantes y pacientes, al igual que Al TakiAmjad, et al., el cual encontró que la minoría de sus pacientes y odontólogos encuestados estaban de acuerdo en que esa proporción era la más atractiva ${ }^{[20]}$; cabe recalcar que, tanto para este estudio como el de Al TakiAmjad, et al., las proporciones más estéticas fueron $71 \%$ y $67 \%$ respectivamente, no concordando así con lo propuesto por Lombardi y Levin ${ }^{[12,20,22]}$. 
Para este parámetro se propone hacer un estudio con un mayor número de variaciones en el ancho del incisivo central superior, siendo así más comparables con otros estudios, y aumentando la especificidad en la elección y percepción de la proporción del ancho entre incisivo central y lateral.

Para la proporción del ancho y largo del incisivo central, la elegida como más atractiva por los tres grupos encuestados fue la de $75 \%$, con una aprobación de $83,3 \%$.

De forma similar Cooper GE, et al. determinó que tanto odontólogos, estudiantes y pacientes perciben de forma más atractiva incisivos con proporciones más cortas, y amplias del $78 \%$ y $82 \%{ }^{[23]}$. En este estudio, no existieron diferencias estadísticamente significativas para la respuesta "atractiva" $(p=0,064)$, a diferencia de los datos encontrados por Cooper GE, et al., en los cuales se observó que para la proporción elegida más atractiva hubo una diferencia estadísticamente significativa entre los estudiantes y pacientes $(p=0,03)^{[23]}$.

La tendencia en los tres grupos encuestados fue de escoger incisivos centrales más cortos y anchos, acercándose a una proporción promedio de la población de $81 \%$ mencionada en el estudio de Moncada GC, et al ${ }^{[8]}$., por lo quese podría plantear que las personas prefieren proporciones similares o coincidentes a la que observan habitualmente en su vida diaria, afectando la etnicidad fuertemente en la aceptación de un tipo de sonrisa en la sociedad.

Los odontólogos consideraron aceptable hasta la proporción de $72 \%$, a diferencia de los estudiantes y pacientes que consideraron aceptable hasta la proporción del $70 \%$, por ende estos últimos dos grupos consideran atractivas un mayor rango de proporciones para el ancho-largo del incisivo central, a diferencia de los odontólogos que fueron más selectivos al momento de escoger una proporción, estos resultados coinciden con el estudio de Wolfart S, et al., ${ }^{[21]}$, pero difiere del estudio de Alsulaimani FF, et al., indicando que, tanto los odontólogos como los pacientes consideran atractivas las mismas proporciones ${ }^{[22]}$.

En este estudio los incisivos centrales más largos, es decir, con proporciones de 64\%, 66\%, $68 \%$ fueron percibidos menos atractivos para la mayoría de los participantes, al igual que en el estudio realizado por Cooper GE, et al., siendo desaprobadas estas proporciones para el 69,8\% de los participantes ${ }^{[23]}$. Se debe mencionar que los pacientes, aunque no eligieron estas proporciones como totalmente atractivas, las calificaron de forma más positiva, a diferencia de los odontólogos y estudiantes que perciben estas proporciones indiscutiblemente como no atractivas. Tal como mencionan Cooper GE, et al., y Wolfart S, et al. ${ }^{[21,23]}$

Estos resultados conducen a que los pacientes son menos detallistas en la apariencia dental con respecto a la proporción entre el ancho y largo del incisivo central y tienen un umbral más alto para percibir las imágenes de forma poco atractivas, prefiriendo una variedad más amplia de proporciones, tanto incisivos cortos como largos.

Como conclusión de este estudio podemos afirmar que no existieron diferencias estadísticamente significativas entre odontólogos, estudiantes de odontología y pacientes para la percepción de los diferentes parámetros estéticos de la sonrisa, a excepción del contorno gingival, en el cual se determinó que los odontólogos son los más sensibles al evaluar este parámetro.

Esta investigación nos hace reflexionar que la estética sigue siendo muy difícil de objetivar, pero se hace necesario intentar estudiar aquellos parámetros que más afectan el resultado final de una sonrisa "atractiva", evitando inducir al paciente a tomar decisiones de tratamiento a partir de lo considerado como poco estético por el odontólogo y no por los propios pacientes, quienes siempre serán únicos y particulares,cuyas percepciones estéticas variarán de acuerdo a los factores socio culturales que los rodean, que siempre tendrán un peso difícil de cuantificar.

\section{REFERENCIAS BIBLIOGRÁFICAS}

1. Lukez A, Pavlic A, Trinajstic ZM, Spalj S. The unique contribution of elements of smile esthetics to psychosocial wellbeing. J Oral Rehabil 2015; 42(4):275-281.

2. Shaw WC, Rees G, Dawe M, Charles CR. The influence of dentofacial appearance on the social attractiveness of young adults. Am J Orthod 1985; 87(1):21-26.

3. Wang C, Hu WJ, Liang LZ, Zhang YL, Chung KH. Esthetics and smile-related characteristics assessed by laypersons. J EsthetRestorDent 2018; 30(2): 136-145.

4. Abu Alhaija ES, Al-Shamsi NO, Al-Khateeb S. Per- 
ceptions of Jordanian laypersons and dental professionals to altered smile aesthetics. Eur J Orthod 2011; 33(4):450-456

5. Al Taki A, Khalesi M, Shagmani M, Yahia I, Al Kaddah F. Perceptions of Altered Smile Esthetics: A Comparative Evaluation in Orthodontists, Dentists, and Laypersons. Int J Dent 2016; 2016: 1-11

6. Chaves ER, Rodriguez J, Peres MFS, Cunningham G. Improving smile and dental esthetics: a comprehensive periodontal and restorative approach after orthodontics. European J Gen Dent 2014; 3(2):170-173.

7. González M, Ibaceta C. Percepción de pacientes y estudiantes de odontología en relación a la estética del sector anterior. Universidad Mayor 2013: 1 - 25.

8. Moncada GC, Angel PA. Parameters for Esthetic Assessment of Upper Anterior Teeth. RevDent Chile 2008; 99(3): 29-38.

9. Del Monte S, Afrashtehfar KI, Emami E, Abi Nader S, Tamimi F. Lay preferences for dentogingival esthetic parameters: A systematic review. J Prosthet Dent 2017; 118(6): 717-724.

10. An SM, Choi SY, Chung YW, Jang TH, Kang KH. Comparing esthetic smile perceptions among laypersons with and without orthodontic treatment experience and dentists. Korean J Orthod 2014; 44(6): 294-303.

11. Rey Mora D, Botero P, Camargo Cruz L, Manejo estético periodontal y ortodóncico del segmento anterior. Revista CES Odontología 2006; vol 19, núm 2.

12. Davis NC. Smile design. Dent Clin North Am 2007; 51(2): 299-318.

13. Tjan AH, Miller GD, The JG. Some esthetic factors in a smile. J ProsthetDent1984; 51(1):24-28.

14. Sterret JD, Oliver T, Robinson F, Fortson W, Knaak B, Russell CM. Width/length ratio of normal clinical crowns of the maxillary anterior dentition in man. J ClinPeriodontol 1999; 26(3): 153-157.

15. Weber B, Fuentes R, García N, Cantín M. Relationships between Shape and Proportion of the Maxillary Central
Incisor with Facial Measurements, Dental and Facial Midline in Adults. International Journal of Morphology 2014; 32(3): 1101-1107.

16. Miller EL, Bodden WR Jr, Jamison HC. A study of the relationship of the Dental Midline to the Facial Midline. J Prosthet Dent 1979; 41(6): 657-660.

17. Pereira F. Percepción, contenido intencional y singularidad. Rev de filosofía 2008; 64: 143-161.

18. Kokich VO, Kokich VG, Kiyak HA. Perceptions of dental professionals and laypersons to altered dental esthetics: Asymmetric and symmetric situations. Am J OrthodDentofacialOrthop 2006; 130(2): 141-151.

19. Geron S, Atalia W. Influence of sex on the perception of oral and smile esthetics with different gingival display and incisal plane inclination. Angle Orthod 2005; 75(5): 778-784.

20. Al Taki A, Handam AM, Mustafa Z, Hassan M, Abu-Alhuda S. Smile esthetics: Impact of variations in the vertical and horizontal dimensions of the maxillary lateral incisors. Eur J Dent 2017; 11(4): 514-520.

21. Wolfart S, Thormann H, Freitag S, Kern M. Assessment of dental appearance following changes in incisor proportions. Eur J Oral Sci 2005; 113(2): 159-165.

22. Alsulaimani FF, Batwa W. Incisors' proportions in smile esthetics JOrthodSci2013; 2(3): 109-112.

23. Cooper GE, Tredwin CJ, Cooper NT, Petrie A, Gill DS. The influence of maxillary central incisor height-to-width ratio on perceived smile aesthetics. Br Dent J 2012; 212(12): 589-599.

\section{RESPONSABLE DEL TRABAJO:}

Nombre: Sofía Bofill Fontboté

Teléfono: +56993301731

Dirección: Av. Libertador Bernardo O’Higgins 2013, Santiago Centro, Región Metropolitana, Chile Correo: sofia.bofill@umayor.cl 